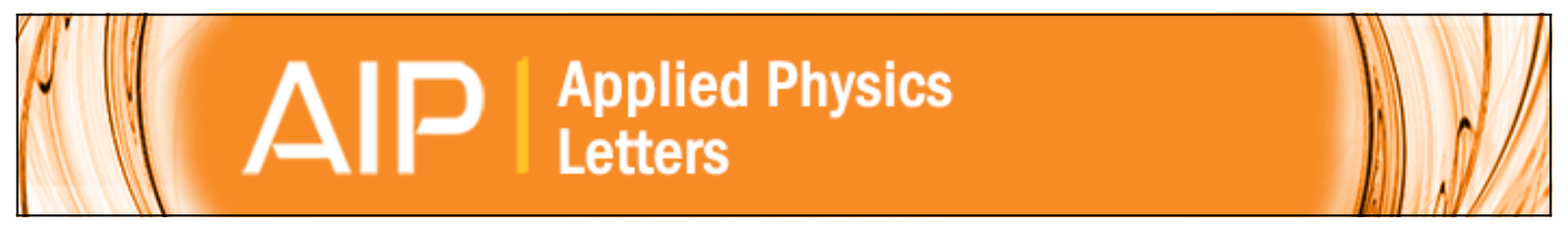

\title{
Ellipsometric characterization of amorphous and polycrystalline silicon films deposited using a single wafer reactor
}

\author{
A. Borghesi, M. E. Giardini, M. Marazzi, A. Sassella, and G. De Santi
}

Citation: Applied Physics Letters 70, 892 (1997); doi: 10.1063/1.118306

View online: http://dx.doi.org/10.1063/1.118306

View Table of Contents: http://scitation.aip.org/content/aip/journal/apl/70/7?ver=pdfcov

Published by the AIP Publishing

\section{Articles you may be interested in}

Electrical and optical properties of sputtered amorphous vanadium oxide thin films

J. Appl. Phys. 111, 073522 (2012); 10.1063/1.3702451

Spectroscopic ellipsometry study of hydrogenated amorphous silicon carbon alloy films deposited by plasma enhanced chemical vapor deposition

J. Appl. Phys. 107, 023502 (2010); 10.1063/1.3277016

Interplay of hydrogen and deposition temperature in optical properties of hot-wire deposited a Si : H Films: Ex situ spectroscopic ellipsometry studies

J. Vac. Sci. Technol. A 23, 1668 (2005); 10.1116/1.2056552

Ex situ spectroscopic ellipsometry and Raman spectroscopy investigations of chemical vapor deposited sulfur incorporated nanocrystalline carbon thin films

J. Appl. Phys. 92, 5457 (2002); 10.1063/1.1511269

Ex situ ellipsometry characterization of excimer laser annealed amorphous silicon thin films grown by low pressure chemical vapor deposition

Appl. Phys. Lett. 71, 359 (1997); 10.1063/1.119537

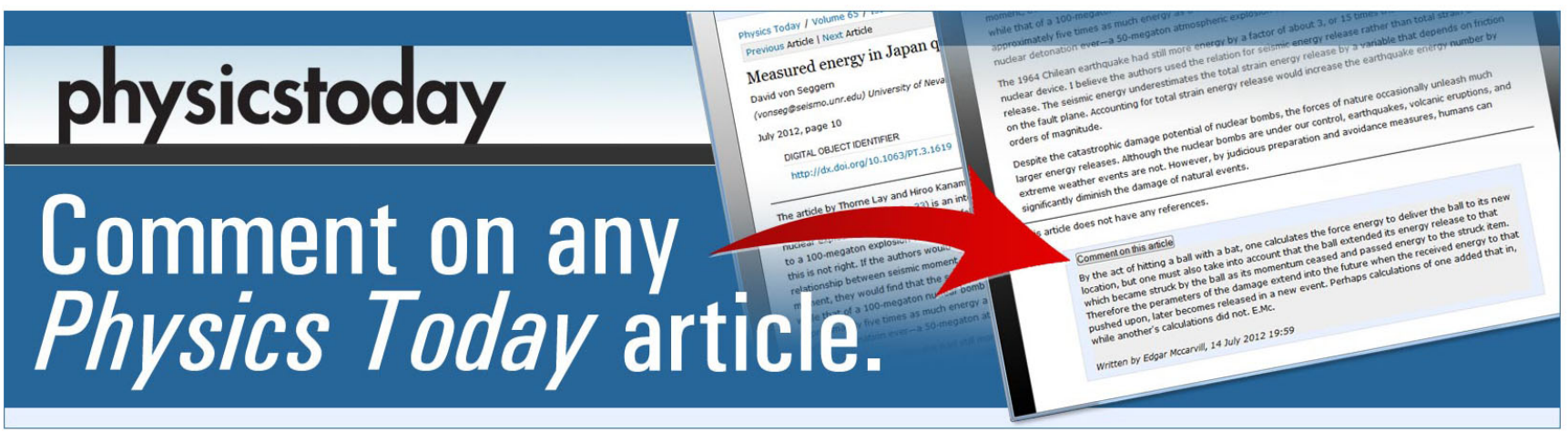




\title{
Ellipsometric characterization of amorphous and polycrystalline silicon films deposited using a single wafer reactor
}

\author{
A. Borghesi \\ Dipartimento di Fisica, Università di Modena, I-41100 Modena, Italy and \\ Laboratorio Materiali e Dispositivi per la Microelettronica of Istituto Nazionale per la Fisica della \\ Materia, Italy \\ M. E. Giardini and M. Marazzi \\ Dipartimento di Fisica “A. Volta,’” Università di Pavia, I-27100 Pavia, Italy and \\ Istituto Nazionale per la Fisica della Materia, Italy \\ A. Sassella $a^{a}$ \\ Dipartimento di Fisica, Università di Milano, I-20133 Milano, Italy and \\ Istituto Nazionale per la Fisica della Materia, Italy \\ G. De Santi \\ SGS-Thomson Microelectronics, I-20041 Agrate B. (MI), Italy
}

(Received 9 July 1996; accepted for publication 15 November 1996)

\begin{abstract}
The optical functions of amorphous and polycrystalline silicon thin films deposited on single oxidized silicon substrates by chemical vapor deposition in a wide range of deposition temperatures have been determined using spectroscopic ellipsometry. The data analysis is performed by direct inversion of the experimental spectra, therefore, obtaining results independent of any film modeling. The optical results indicate that the film structure changes as the deposition temperature increases from amorphous to polycrystalline with different grain size and distribution. (C) 1997 American Institute of Physics. [S0003-6951(97)02203-1]
\end{abstract}

Polycrystalline silicon $(p-\mathrm{Si})$ is certainly acknowledged as a key material in electronic device manufacturing. Recently, a new generation of deposition equipment has been developed, where $p$-Si films are deposited by single wafer chemical vapor deposition (CVD). These systems offer peculiar properties of uniformity on wafer and wafer-to-wafer reproducibility in spite of the fact that deposition occurs at a very high rate (about $50-100 \mathrm{~nm} / \mathrm{min}$ ). This technology is properly extendible to the application in the Gbit generation devices. A deep characterization of the films deposited under different conditions is necessary for a complete knowledge of the $p$-Si properties and of the deposition reactors and for the tuning of reliable processes for the different applications. Among the different characterization techniques, the optical ones are the most interesting, due to their nondestructiveness, high sensitivity, and in situ applicability without any influence on the process conditions.

In this letter, a spectroscopic ellipsometry study of phosphorus-doped $p$-Si films deposited by single wafer CVD in a wide range of deposition temperatures is reported. The complex dielectric function $\widetilde{\epsilon}$ of the different films is obtained and a close correlation between $\widetilde{\epsilon}$ and the structural properties of the samples is found.

Twelve $P$-doped $\left(2 \times 10^{20} P\right.$ atoms $\left./ \mathrm{cm}^{3}\right) \quad p$-Si films were deposited from diluted $\mathrm{SiH}_{4}$ and $\mathrm{PH}_{3}$ using an apparatus HT Centura by Applied Materials on a 80-nm-thick thermal oxide film on (100)-oriented Czochralski silicon substrates. Each film was deposited in $150 \mathrm{~s}$. After the deposition, the samples were stored in air, therefore, allowing the growth of a few-nm-thick native $\mathrm{SiO}_{2}$ layers to occur. This series of samples has been deposited varying the substrate temperature from 605 to $660{ }^{\circ} \mathrm{C}$ in $5^{\circ} \mathrm{C}$ steps (samples

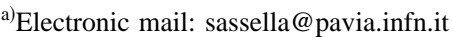

$1-12)$. Ellipsometry measurements were carried out in the spectral range from 1.5 to $5 \mathrm{eV}(0.25-0.85 \mu \mathrm{m})$ using a rotating polarizer instrument SOPRA ES 4G, with $2 \mathrm{~nm}$ spectral resolution. The ellipsometric experimental data are the $\tan \psi$ and $\cos \Delta$ functions, ${ }^{1}$ as defined from

$$
\tilde{\rho}=\frac{\widetilde{r_{p}}}{\widetilde{r_{s}}}=\tan \psi \exp i \Delta,
$$

where $\widetilde{r_{p}}$ and $\widetilde{r_{s}}$ are the Fresnel reflection coefficients for $p$-polarized and $s$-polarized light, respectively.

In Fig. 1 typical experimental spectra of $\tan \psi$ and $\cos \Delta$ for a $p$-Si sample (sample 9 deposited at $645^{\circ} \mathrm{C}$ ) are shown. Considering the penetration depth of the visible light in amorphous silicon $(a-\mathrm{Si})$ and in single-crystal silicon and assuming that for all the $p$-Si samples the penetration depth lies between those of such two reference materials, in the spectra of Fig. 1 two regions can be distinguished. ${ }^{2}$ In the high-energy region, i.e., from about 3 to $5 \mathrm{eV}$, the light penetration depth is about $10 \mathrm{~nm}$ therefore, only the surface native oxide covering the $p$-Si film and its interface with $p$-Si are investigated. In this region, the fitting procedures (discussed below) used to interpret the bare spectra give satisfactory results for samples 1-9 (dots in Fig. 1 are the best fit data of sample 9), suggesting a negligible effect of the roughness of the interface between the native $\mathrm{SiO}_{2}$ and the $p$-Si layer, possibly present. On the contrary, the experimental data for the high-temperature samples (from 10-12) are affected by the surface roughness, which increases as the deposition temperature increases, as evidenced by the results of the fits. A quantitative evaluation of such a roughness obtained by atomic force microscopy (AFM) measurements gives a root-mean-square value varying from about 1 to about $5 \mathrm{~nm}$ from sample 1 to 12 . In the spectral range from 1.5 to about $3 \mathrm{eV}$ the light penetrates the $p$-Si and the thermal oxide layers, reaching the interface with the silicon sub- 


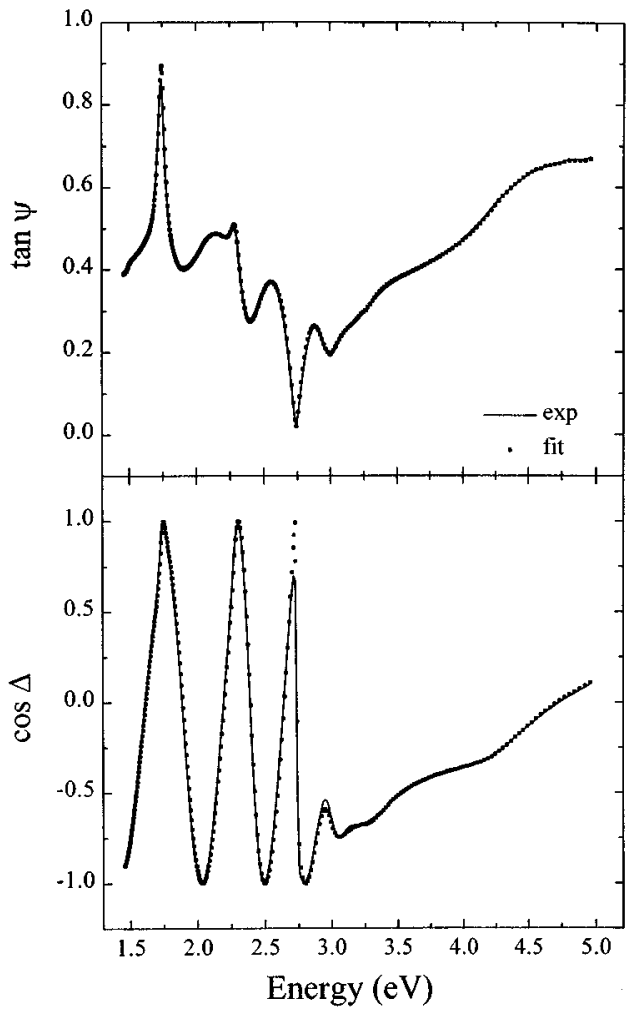

FIG. 1. Experimental tan $\Psi$ and $\cos \Delta$ spectra of sample 9 (full lines) and best fit (dots).

strate. In this region, the experimental spectra of all samples are very similar to each other, showing strong interference fringes related to both the $p$-Si and the thermal oxide layers. Around $3 \mathrm{eV}$ the fitting of experimental data is not very good due to the well-known poor quality of the $p$-Si/thermal oxide interface for deposition temperatures higher than about $615^{\circ} \mathrm{C} .^{3}$ For lower energy the fitting of the experimental data is much easier, probably because of good and constant $\mathrm{SiO}_{2}$ stoichiometry and structural order.

All the samples were modeled using the same three-layer structure, i.e. (from surface to substrate), a native oxide layer, the $p$-Si film of unknown thickness and optical properties, a thermal $\mathrm{SiO}_{2}$ layer of $80 \mathrm{~nm}$, and the single-crystal silicon substrate. The possibility of describing $p$-Si by a mixture of $a-\mathrm{Si}$ and single-crystal silicon, as far as it regards optical properties, is debatable and this point is discussed in the literature. ${ }^{4,5}$ In our opinion, as a first approach aimed at obtaining qualitative information on $p$-Si film crystallinity, a regression of the experimental $\tan \psi$ and $\cos \Delta$ considering the $p$-Si layer as a Bruggeman effective medium ${ }^{6}$ of the literature $a$-Si and single-crystal silicon ${ }^{7}$ can fruitfully be performed. In particular, for all the samples the thickness of the native oxide layer was determined by fitting the experimental curves in the high-energy region, while those of the $p$-Si and of the thermal oxide layers were determined in the low-energy region. Then, the fitting procedure over the whole spectral range was iterated several times till convergence was achieved. Both a low standard deviation ${ }^{8} \sigma$ and low correlation coefficients between the fitting parameters were used as criteria for selecting the best fit.

The results of this first regression, reported in Table I as a percentage of $a$-Si in the effective medium approximation
TABLE I. Amorphous silicon content in the $p$-Si layers resulting from the first regression of experimental ellipsometric data, and layer thickness $t$ and standard deviation $\sigma$ obtained after regression refinement and iteration.

\begin{tabular}{lrrc}
\hline \hline Sample & $a$-Si $(\%)$ & $t(\mathrm{~nm})$ & $\sigma \times 10^{2}$ \\
\hline $1\left(605^{\circ} \mathrm{C}\right)$ & 100.0 & 65.8 & $\ldots .^{\mathrm{a}}$ \\
$2\left(610^{\circ} \mathrm{C}\right)$ & 100.0 & 77.5 & 0.62 \\
$3\left(615^{\circ} \mathrm{C}\right)$ & 99.0 & 89.0 & 0.63 \\
$4\left(620^{\circ} \mathrm{C}\right)$ & 94.8 & 101.1 & 1.00 \\
$5\left(625^{\circ} \mathrm{C}\right)$ & 81.6 & 113.5 & 2.86 \\
$6\left(630^{\circ} \mathrm{C}\right)$ & 28.2 & 130.7 & 3.23 \\
$7\left(635^{\circ} \mathrm{C}\right)$ & 16.3 & 147.9 & 1.36 \\
$8\left(640^{\circ} \mathrm{C}\right)$ & 13.2 & 170.8 & 0.99 \\
$9\left(645^{\circ} \mathrm{C}\right)$ & 16.9 & 200.8 & $\ldots 0^{\mathrm{a}}$ \\
$10\left(650^{\circ} \mathrm{C}\right)$ & 14.2 & 238.1 & 1.62 \\
$11\left(655^{\circ} \mathrm{C}\right)$ & 16.8 & 282.3 & 1.70 \\
$12\left(660^{\circ} \mathrm{C}\right)$ & 15.9 & 328.7 & 2.19 \\
\hline \hline
\end{tabular}

${ }^{\text {a }}$ Reference sample.

(EMA) mixture, clearly show that sample 1 can be considered as fully amorphous and that in samples 8-12 the percentage of $a$-Si is the same. This suggests the possibility of employing the optical functions of the $p$-Si layer of sample 1 (amorphous) and of the $p$-Si layer of a sample among 9-12 as internal references to repeat the regression obtaining more reliable thickness values. As reference polycrystalline material, we chose $p$-Si of sample 9 because atomic force microscopy measurements show that it is affected by the lowest surface roughness among samples 9-12. As a starting step, we supposed that sample 1 and sample 9 are composed of $a-\mathrm{Si}$ and $p-\mathrm{Si}$, respectively, which we expect to be similar to the ones reported in Ref. 3, deposited by conventional CVD. Therefore, a second regression only on these samples was performed using the same EMA description as before, but considering a mixture of these two literature ${ }^{4}$ materials and single-crystal silicon, obtaining reliable thickness values. These data, as well as the thickness of the thermal $\mathrm{SiO}_{2}$ layers, are in agreement with transmission electron microscopy (TEM) results within 3\%. We then abandoned the EMA description of the $p$-Si layer because, once the layer thickness is known, a direct numerical inversion of the ellipsometric data is possible and gives more reliable spectra of the complex dielectric function $\tilde{\epsilon}=\epsilon_{1}+i \epsilon_{2}$, being free of any film modeling.

The optical functions $\epsilon_{1}$ and $\epsilon_{2}$ obtained for the amorphous $p$-Si layer in sample 1 are very similar to those of $a$-Si deposited by conventional CVD, while the functions of the $p$-Si layer in sample 9 are strongly different from those of $p$-Si deposited by the conventional CVD techniques.

To evaluate the complex dielectric function of all the other $p$-Si layers, a procedure for data analysis similar to that used for samples 1 and 9 was adopted, considering as reference data the $\widetilde{\epsilon}$ of these two samples. First, data regression has been refined describing the $p$-Si layer as an EMA mixture to obtain reliable thickness values. The agreement found with TEM results is again very good (within 3\%-4\%). Then, the $\epsilon_{1}$ and $\epsilon_{2}$ functions were calculated by numerical inversion of the ellipsometric data. In particular, to obtain the film thickness the $p$-Si layer in sample 2 was modeled by a mixture of the $p$-Si of sample 1 and crystalline silicon, the $p$-Si layer in sample 3 was modeled by a mixture of $p$-Si of 


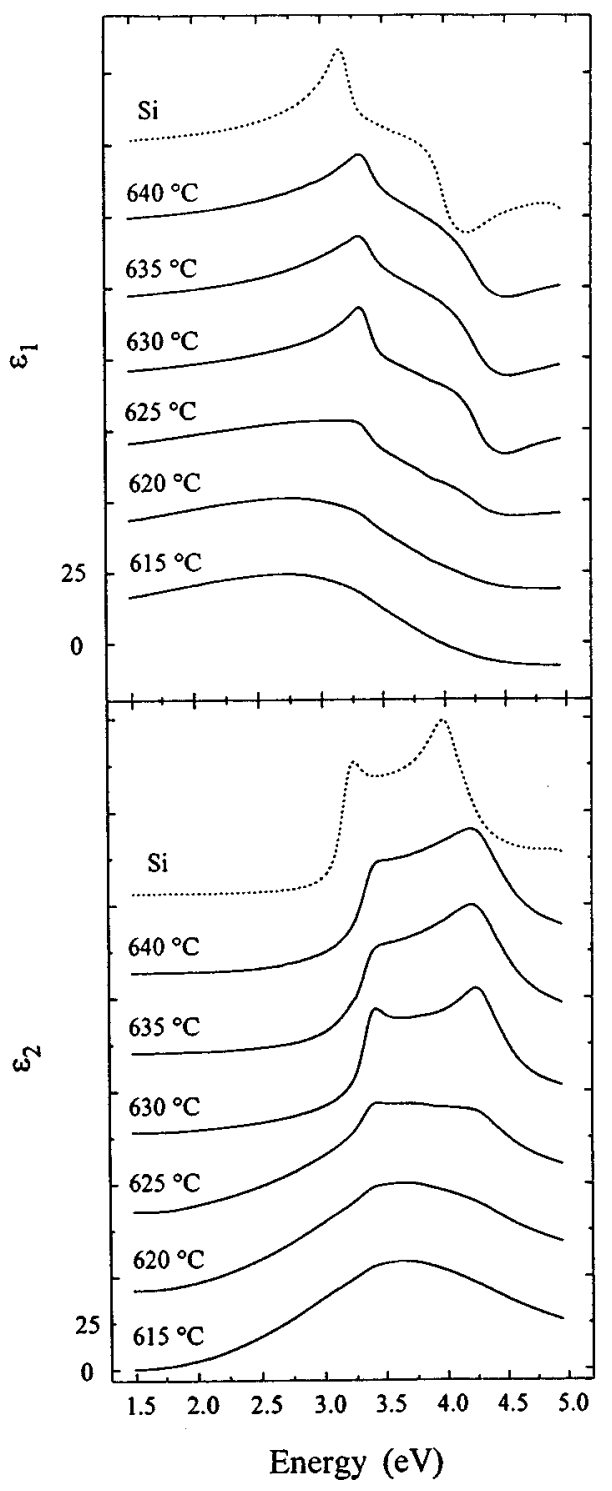

FIG. 2. Real and imaginary parts $\epsilon_{1}$ and $\epsilon_{2}$ of the complex dielectric functions of samples from 3 to 8 (deposition temperature from 615 to $640{ }^{\circ} \mathrm{C}$, full lines) and of literature single-crystal silicon (dashed lines). The vertical scales refer to the $615^{\circ} \mathrm{C}$ spectra; each of the other $\epsilon_{1}$ and $\epsilon_{2}$ spectra has been shifted by 30 and 40 units, respectively, with respect to the preceeding one.

sample 2 and crystalline silicon, and so on for sample 4. A similar procedure for the analysis of the experimental data of samples 8 to 5 was adopted, considering the optical functions of the $p$-Si layer in sample 9. More precisely, such optical functions together with $a$-Si and crystalline silicon functions were used for the EMA modeling of the $p$-Si layer in sample 8 to get its thickness, and so on down to sample 5, obtaining very good results. The $\widetilde{\epsilon}$ function for the high-temperature $p$-Si films (samples 10-12) were also derived in the same way, starting from the reference $\widetilde{\boldsymbol{\epsilon}}$ of $p$-Si in sample 9 . The layer thickness $t$ and the parameter $\sigma$ in Table I are the $p$-Si thickness and standard deviation resulting from such analysis.

A low standard deviation $\sigma$ is reached for samples 1-3 and 7-11, deposited at the lowest and the highest temperatures, respectively, while $\sigma$ is less satisfactory for samples 5, 6 , and 7. As for the $p$-Si layers in the samples, TEM images show that they are dispersions of isolated crystalline grains in an amorphous matrix. Such grains are larger as the deposition temperature is higher, i.e., from sample 5-7, with dimensions of the order of the wavelength. Therefore, for such samples the EMA is very poor and this justifies the high standard deviation reported in Table I. The high value of $\sigma$ for sample 12 has to be attributed to the mentioned surface roughness.

Figure 2 shows the spectra of $\epsilon_{1}$ and $\epsilon_{2}$ for samples 3-8, deposited at temperatures from 615 to $640{ }^{\circ} \mathrm{C}$ (full lines), obtained from the numerical inversion of the ellipsometric data, and for literature crystalline silicon (dashed lines). The results for the fully amorphous samples (1 and 2) and for the polycrystalline ones (9-12) are not reported, being exactly the same as those for samples 3 and 8 , respectively.

The shape of the $\epsilon_{1}$ and $\epsilon_{2}$ curves changes from that of $a-\mathrm{Si}$ to very close to that of crystalline $\mathrm{Si}$ as the deposition temperature increases from 615 to $630{ }^{\circ} \mathrm{C}$. The optical behavior of the $p$-Si deposited at this last temperature is the most similar to that of single-crystal silicon. Indeed, the intensity of the peaks in $\epsilon_{1}$ and $\epsilon_{2}$ at about 3.3 and $4.5 \mathrm{eV}$ for the samples deposited at higher temperatures is lower. These peaks are indicative of the crystallinity of the samples, being related to critical points in the joint density of states of single-crystal silicon, ${ }^{9,10}$ and are very sensitive to the size of the crystals. Therefore, the height of these peaks in the spectra of the sample deposited at $630{ }^{\circ} \mathrm{C}$ indicate that it contains the largest crystalline grains.

In conclusion, the optical functions of $P$-doped polycrystalline silicon films deposited by CVD were obtained for a wide range of deposition temperatures from spectroscopic ellipsometry measurements by direct inversion of the experimental data, therefore, independently of any modeling of the film. The low-temperature films are amorphous and very similar to conventional CVD $a$-Si, while the hightemperature ones are polycrystalline with a strongly different optical behavior with respect to conventional CVD $p$-Si films. A transition temperature was observed in the range from 625 to $640{ }^{\circ} \mathrm{C}$, where the $p$-Si structure shifts from that of a crystalline grain dispersion in an $a$-Si matrix to that of a true polycrystal, with closely packed grains.

${ }^{1}$ D. E. Aspnes, in Handbook of Optical Constants of Solids, edited by E. D. Palik (Academic, Orlando, 1985), p. 89.

${ }^{2}$ L. M. Asinovsky, Thin Solid Films 233, 210 (1993).

${ }^{3}$ F. Hottier and R. Cadoret, J. Cryst. Growth 56, 304 (1982).

${ }^{4}$ G. E. Jellison, Jr., M. F. Chisholm, and S. M. Gorbatkin, Appl. Phys. Lett. 62, 3348 (1993).

${ }^{5}$ Y. Z. Hu, D. J. Diehl, C. Y. Zhao, C. L. Wang, Q. Liu, E. A. Irene, K. N. Christensen, D. Venable, and D. M. Maher, J. Vac. Sci. Technol. B 14, 744 (1996).

${ }^{6}$ D. A. G. Bruggeman, Ann. Phys. (Leipzig) 24, 636 (1935).

${ }^{7}$ G. E. Jellison, Jr., Opt. Mater. 1, 41 (1992).

${ }^{8}$ See for example, P. R. Bevington, Data Reduction and Error Analysis for the Physical Sciences (McGraw-Hill, New York, 1969).

${ }^{9}$ A. Daunois and D. E. Aspnes, Phys. Rev. B 18, 1824 (1978).

${ }^{10}$ P. Lautenschlager, M. Garriga, L. Viña, and M. Cardona, Phys. Rev. B 36, 4821 (1987). 